\title{
Choroidal Paraganglioma with Metastases to the Fellow Eye
}

\author{
Rita Van Ginderdeuren ${ }^{a, b}$ Guy S. Missotten ${ }^{a}$ Joost van den Oord ${ }^{b}$ \\ Departments of ${ }^{a}$ Ophthalmology and ${ }^{b}$ Pathology, University Hospitals Leuven, \\ Leuven, Belgium
}

\section{Key Words}

Choroidal tumor · Metastatic disease $\cdot$ Paraganglioma

\begin{abstract}
Purpose: To report a case of a paraganglioma in the right eye with metastatic disease in the fellow eye 3 years later. Methods: A 70-year-old man presented with a painful amblyopic right eye; rubeosis iridis and a large choroidal tumor were found. The tumor was treated by enucleation. Pathology diagnosed the tumor as a paraganglioma. Screening for other tumors or metastatic disease was negative at that moment. After 3 years, a paraganglioma skin metastasis was detected, and screening revealed metastatic disease in the liver. Another 6 months later he was referred for tumors in the left eye, which were treated by radiotherapy. He succumbed 6 months later. Results: Histopathology of the right eye revealed the typical image of a paraganglioma, with expression of synaptophysin, neuron-specific enolase and chromogranin. S-100 staining was positive in the sustentacular cells; staining for HMB-45, SME, EMA and pan-keratin was negative. Microscopy of the tumors in the skin and liver 3 years later showed a dedifferentiated tumor with the same immunological characteristics, but with higher Ki67 expression and more mitoses. Conclusions: This report documents a very rare choroidal paraganglioma which presented clinically as a melanoma. The patient succumbed 4 years later to generalized metastatic disease. No other primary paraganglioma was found; however, paraganglion cells in the eye have never been described.
\end{abstract}

\section{Introduction}

A paraganglioma is a very rare tumor originating from the cells of the paraganglia $[1,2]$. It is usually found around the spinal cord and in the neck region [3]. Location in the head and orbit is very rare, and in the eye it has only been described twice [4-6]. Usually, a paragan- 
glioma runs an uneventful course but recurrences and metastatic disease may rarely occur and are difficult to treat because of lack of specific treatment [1].

\section{Case Report}

A 70-year-old man presented with a painful and red amblyopic right eye. His vision was hand movements right and 10/10 left. Biomicroscopy showed rubeosis iridis and a retrolenticular mass; fundus examination revealed a huge non-pigmented choroidal tumor and retinal detachment. Ultrasonography demonstrated a large mushroom-shaped intraocular tumor, originating in the choroid with total retinal detachment. There was medium reflectivity with attenuation. Screening for metastatic disease with MRI and CT of the abdomen, brain and thorax, was negative. The right eye was enucleated. Histopathological examination showed a non-pigmented tumor of spindle-shaped and epithelioid cells arranged in nests; the cells were negative for Melan-A and positive for neuroendocrine markers. The diagnosis was paraganglioma. Follow-up examination 3 years later demonstrated a metastatic location of paraganglioma in the skin. One year later, identical tumors were found in the prostate and liver. The patient was referred to our department for tumors in the left eye. His vision was $7 / 10$ with metamorphopsia. Fundoscopy revealed 4 elevated, choroidal non-pigmented lesions (fig. 1). OCT demonstrated submacular fluid. Ultrasound examination showed a tumor with medium reflectivity and no attenuation, of $6 \mathrm{~mm}$ height and $12 \mathrm{~mm}$ thickness. The eye was irradiated, resulting in diminishment of the fluid. The patient succumbed 9 months later due to generalized disease with multiple metastases and infectious complications. He had no clinical manifestations of catecholamine-induced changes such as hypertension or cardiac arrhythmias, and laboratory examination of blood and urine were negative for markers of neuroendocrine tumors. There were no familial antecedents of pheochromocytoma, paraganglioma or neuroendocrine disease. An autopsy was, however, refused by the family and is not a common procedure or legal obligation in our country.

\section{Histopathological Analysis}

The first biopsy (2006), of the right eye, revealed a large choroidal tumor, consisting of nests of medium-sized spindle- and epithelioid-shaped cells. These cells were separated by fibrovascular septa and assumed the typical image of 'Zellballen' (fig. 2). The cytoplasm was focally eosinophilic or pale without pigmentation. Few mitoses were recognized (less than 1 per 20 high-field images). There was discrete cellular and nuclear pleomorphism. Immunostaining was negative for HMB-45, Melan-A, SMA, EMA and pancytokeratin, and positive for synaptophysin, neuron-specific enolase and chromogranin; S-100 staining was only positive in the surrounding (sustentacular) cells of the cell clusters (fig. 3). The image shown in fig. 3 is typical of a paraganglioma. Ki67 was positive in less than $10 \%$ of tumor cell nuclei.

The biopsy of the skin lesion (2009) showed a normal epidermis and within the dermis there were multiple nests of pale epithelioid cells with atypia and rare mitoses. The typical Zellballen configuration was present in only a small region. The results of immunostaining were identical to those performed for the eye tumor. Ki67 was positive in more than $10 \%$ of cells.

Later biopsies of the liver and prostate (2009) revealed identical cell nests, with more pleomorphism, a higher mitotic rate (1-4 per 20 high-field images) and no typical Zellballen pattern; these lesions showed a more diffuse architecture with mostly very pale cells. In the 
prostate, loss of normal glandular tissue was observed. The results of immunostaining were identical, but with less obvious $\mathrm{S}-100$ positivity.

The tumors in the left eye were not biopsied.

\section{Discussion}

This patient had a very rare manifestation of non-secreting paraganglioma, which presented first in one eye; 3 years later he had metastases to the skin, and 6 months later in the fellow eye. He succumbed to generalized metastatic disease.

A paraganglioma is a rare tumor which originates from paraganglia cells at various body sites [1, 2]. These cells originate from embryonic neural crest cells and belong to the group of neuroendocrine cells dispersed throughout the body and connected with the autonomic nervous system. The largest collection of these cells is found in the adrenal medulla where they give rise to pheochromocytomas. Distinct ganglia are localized around the spinal cord and the cranial nerves. The current World Health Organization classification (2004) reserves the term pheochromocytoma for intra-adrenal tumors, whereas similar tumors at other locations are defined as extra-adrenal paragangliomas. Paragangliomas arise from chromaffin cells in sympathoadrenal and parasympathetic paraganglia. These cells normally act as special chemoreceptors located along blood vessels, particularly in the carotid and aortic bodies. Paragangliomas belong to the larger category of neuroendocrine tumors, and the histological picture of this eye tumor is morphologically very typical for a paraganglioma; the immunostaining is similar to other neuroendocrine tumors or carcinoids, but with negative markers for keratin $[7,8]$. This patient did not have any symptoms of a carcinoid or neuroendocrine tumor. CT and MRI of the abdomen and thorax, and biochemical parameters in plasma were negative for other locations during 3 years. Neuroendocrine tumors can be functioning or not (i.e. secreting hormones or not), in which case clinical presentation may differ [2].

Paragangliomas form characteristically small nests of uniform polygonal chromaffin cells (Zellballen). These cell balls are separated by fibrovascular stroma and surrounded by sustentacular cells. They are highly vascularized tumors. A diagnosis of malignancy can only be made by identifying tumor deposits in tissues that do not normally contain chromaffin cells [1]. Pathological features associated with future malignant behavior include large tumor size, increased number of mitoses, DNA aneuploidy and extensive necrosis [1, 2]. Pathology generally provides insufficient prognostic information regarding the likelihood of recurrence or metastasis $[1,2]$. There is no standard staging system for paragangliomas. There are no clear data regarding the survival of patients with localized disease or regional disease; $6.5-16.5 \%$ of these patients will develop a recurrence, usually 5-15 years after initial surgery. Approximately 50\% of patients with recurrent disease experience distant metastasis. The 5-year survival in cases of metastatic disease is $40-45 \%$ [2]. Surgery is the mainstay of treatment for most patients. Patients require lifelong monitoring for disease recurrence $[1,2]$.

About $75 \%$ of paragangliomas are sporadic and the remaining $25 \%$ are hereditary; the presentation in familial cases is typically between the 2nd and 3rd decade and with multifocal paragangliomas. Mutations of the genes SDHD, PGL2, SDHC and also MEN have been identified as involved in paragangliomas [9]. This patient had no familial antecedents and the older age at presentation (70 years) is in favor of a sporadic tumor.

A paraganglioma in the eye is very rare; it has been described in 1 case in the iris as a single location with a benign outcome [5] and in another case as metastasis [6]. Paraganglia 
cells have never been described in the eye and hence, it is not known whether this type of cells exists at that location. Sandboe et al. [5], in their case of primary iris paraganglioma, suggested the possibility that there are stretch receptors in the iris, resembling the known stretch receptors around the aortic arch which are paraganglia cells. The possibility of a primary tumor elsewhere was investigated but not found by full body scans (PET, MRI and $\mathrm{CT}$ ). All the non-ocular tumors found 3 years after the primary presentation in the right eye were obviously less differentiated and showed more atypia and a higher mitotic index, which is the typical progression of metastatic disease. There is no specific treatment known for this rare disease: surgery can be radical, but once metastatic disease occurs, only palliative radiotherapy and chemotherapy can be offered.

\section{Disclosure Statement}

None of the authors has a conflict of interest disclose.

No financial support was received for this submission.

This study was performed with informed consent.

\section{References}

1 Tischler AS, Kimura N, McNicol AM: Pathology of pheochromocytoma and extra-adrenal paraganglioma. Ann NY Acad Sci 2006;1073:557-570.

-2 McNicol AM: Update on tumours of the adrenal cortex, phaeochromocytoma and extra-adrenal paraganglioma. Histopathology 2011;58:155-168.

-3 Pellitteri PK, Rinaldo A, Myssiorek D, et al: Paragangliomas of the head and neck. Oral Oncol 2004;40:563575.

-4 Sharma MC, Epari S, Gaikwad S, Verma A, Sarkar C: Orbital paraganglioma; report of a rare case. Can J Ophthamol 2005;40:640-644.

5 Sandboe FD, Elgjo K, Eide N, Medin W, Scott H: An intraocular paraganglioma? Acta Opthalmol 1994;72:138-141.

6 Schalenbourg A, Moulin A, Guillou L, Zografos L: Metastatic choroidal paraganglioma. Opthalmology 2011;118:2238-2241.

7 Riddle PJ, Font RL, Zimmerman LE: Carcinoid tumors of the eye and orbit: a clinicopathologic study of 15 cases, with histochemical and electron microscopic observations. Hum Pathol 1982;13:459-469.

8 Buettner H, Bartley GB, Bolling JP: Clinical features and treatment of seven patients with carcinoid tumor metastatic to the eye and orbit. Am J Ophthalmol 1995;119:211-218.

9 Schipper J, Boedeker C, Maier W, Neumann H: Paragangliomas in the head-/neck region. I. Classification and diagnosis (in German). HNO 2004;52:569-574, quiz 575. 
Case Reports in

Ophthalmology

\begin{tabular}{l|l}
\hline Case Rep Ophthalmol 2013:4:17-22 \\
\hline DOI: $\underline{10.1159 / 000347169}$ & $\begin{array}{l}\text { C 2013 S. Karger AG, Basel } \\
\text { www.karger.com/cop }\end{array}$ \\
\hline
\end{tabular}

Van Ginderdeuren et al.: Choroidal Paraganglioma with Metastases to the Fellow Eye

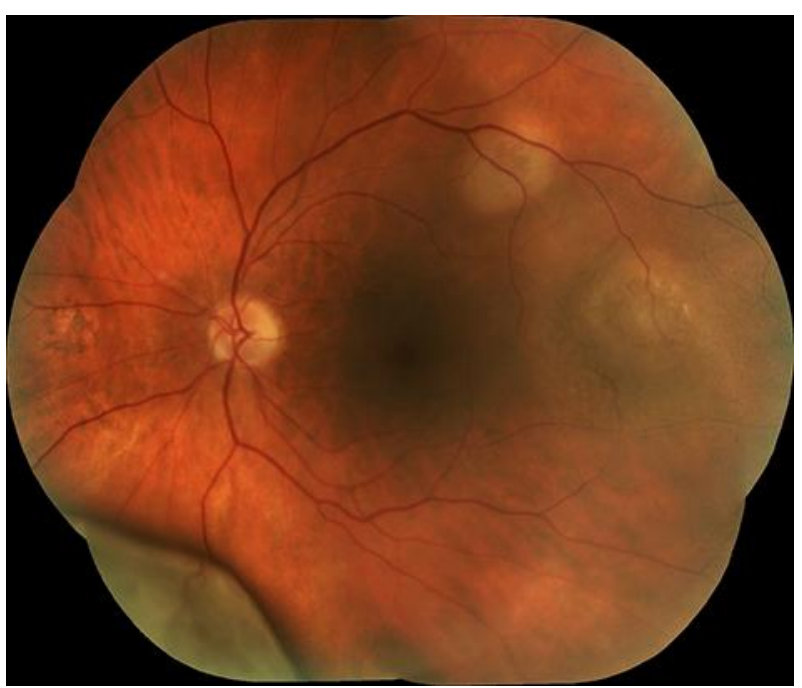

Fig. 1. Fundus picture of choroidal tumors in the left eye.

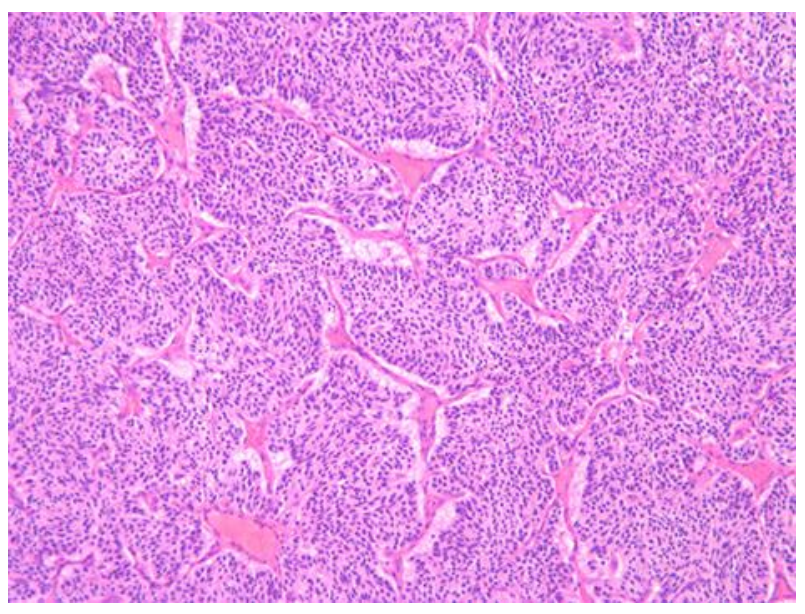

Fig. 2. Microphotography of the paraganglioma in the right eye showing nests of spindle-shaped cells, surrounded by fibrovascular septa, typical Zellballen (HE, $\times 10$ objective). 


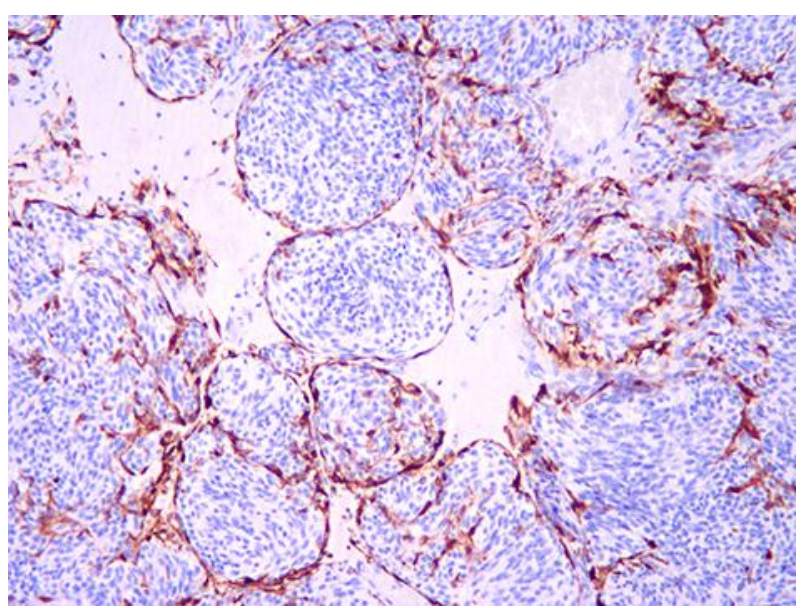

Fig. 3. S-100 staining of the sustentacular cells on the periphery of the tumor nests $(\times 10$ objective). 